\title{
STUDY OF CHARGE MULTIPLICITY IN HADRONIC THREE-JET Z DECAYS AT LEP *
}

\author{
VLADIMIR A. UVAROV \\ Institute for High Energy Physics, Protvino, Russia \\ (for the DELPHI Collaboration)
}

\begin{abstract}
The mean charge multiplicity in hadronic three-jet $\mathrm{Z}$ decays has been measured with the DELPHI detector as a function of the event topology and compared with recent theoretical calculations. The QCD colour factor ratio $C_{A} / C_{F}$ was determined, and the gluon contribution to this multiplicity was extracted giving a measurement of the mean charge multiplicity of a two-gluon colour-singlet system as a function of the effective c.m. energy covering the range from 16 to $52 \mathrm{GeV}$.
\end{abstract}

The radiation of gluons by quarks (other gluons) is proportional to the colour charge $C_{F}\left(C_{A}\right)$, where $C_{F}$ and $C_{A}$ are the eigenvalues of the quadratic Casimir-operators of the $S U(3)_{C}$ group. Therefore, the comparison of the contributions of quark and gluon jets to the mean particle multiplicity in hadronic $\mathrm{Z}$ decays gives insight into the group structure of the QCD. These contributions can be studied within the framework of the colour dipole model ${ }^{1,2}$ which predicts the mean charge multiplicity of hadronic three-jet $e^{+} e^{-}$events as a function of the event topology. Recently this model has been applied ${ }^{3,4}$ to investigate the multiplicity of symmetric $\mathrm{Z} \rightarrow q \bar{q} g$ events only with one relevant scale. To provide a more thorough test of this model, in the present study, ${ }^{5}$ results of which are preliminary, the multiplicity is measured for asymmetric $\mathrm{Z} \rightarrow q \bar{q} g$ events as a function of two relevant scales, then the colour factor ratio $C_{A} / C_{F}$ and the gluon contribution to this multiplicity are determined.

The data used in this analysis have been collected with the DELPHI detector at $\sqrt{s}=M_{Z}$ in the years 1992-95. The reconstruction and selection criteria of charged and neutral particles and hadronic events have been described in Ref. ${ }^{6}$. All selected particles in each selected event were clustered to a three-jet configuration using the Cambridge,$^{7}$ the angular ordered Durham ${ }^{7}$ and the Durham ${ }^{8}$ algorithms without a specified value of the jet resolution parameter $y_{\text {cut }}$. The correction factor for the detector acceptance and inefficiencies calculated from the Monte Carlo simulation

*Talk given at the 32nd International Symposium on Multiparticle Dynamics, Alushta, Ukraine, September 7-13, 2002. 
as a function of the event topology was applied to the data.

The topology of the event with three massless jets can be described by two of three inter-jet angles numbered in order of size $\left(\theta_{1}<\theta_{2}<\theta_{3}\right)$. As $y_{\text {cut }}$ is adjusted separately for each event, only that $\left(\theta_{3}, \theta_{2}\right)$ range is used in the analysis where the mean value of $\log _{10}\left(y_{2} / y_{3}\right)$ is greater than 1 for each data point (here $y_{n}$ is the smallest test variable $y_{i j}$ in an event at the stage of clustering when $n+1$ jets are still present). Also the $\left(\theta_{3}, \theta_{2}\right)$ range where the correction factor deviates from a smooth behaviour or the contribution to $\chi^{2}$ of the fit is too large is excluded from the analysis. Finally, the chosen intervals are the following: $120^{\circ}<\theta_{3}<155^{\circ}$ and $108^{\circ}<\theta_{2}<155^{\circ}$.

The colour dipole model ${ }^{1}$ predicts the relationship between the scale evolution of the mean charge multiplicities of two-gluon, $N_{g g}(L)$, and quarkantiquark, $N_{q \bar{q}}(L)$, colour-singlet systems:

$$
\frac{d}{d L} N_{g g}\left(L+c_{g}-c_{q}\right)=\left(C_{A} / C_{F}\right) \cdot \frac{d}{d L} N_{q \bar{q}}(L) \cdot\left(1-\alpha_{0} c_{r} / L\right),
$$

where $L=\ln \left(s / \Lambda^{2}\right), s=E_{c . m}^{2}, \Lambda=250 \mathrm{MeV}, c_{g}=11 / 6, c_{q}=3 / 2$, $c_{r}=10 \pi^{2} / 27-3 / 2, \alpha_{0}=6 C_{A} /\left(11 C_{A}-2 N_{f}\right)$ and $N_{f}=5$. The constant of integration of this differential equation has been fixed by the CLEO measurement ${ }^{9}$ of the mean charge multiplicity in $\chi_{b}^{\prime}(J=2) \rightarrow g g$ decays.

The mean charge multiplicity, $N_{q \bar{q} g}$, of hadronic three-jet $e^{+} e^{-}$events defined by a $k_{\perp}$-based jet finder without a specified resolution scale is given by two alternative expressions ${ }^{2}$ with a single scale $k_{\perp}$ for the gluon jet:

$$
\begin{aligned}
& N_{q \bar{q} g}=N_{q \bar{q}}\left(L_{q \bar{q}}, k_{\perp L u}\right)+\frac{1}{2} N_{g g}\left(k_{\perp L e}\right), \\
& N_{q \bar{q} g}=N_{q \bar{q}}\left(L, k_{\perp L u}\right)+\frac{1}{2} N_{g g}\left(k_{\perp L u}\right),
\end{aligned}
$$

where $L_{q \bar{q}}=\ln \left(s_{q \bar{q}} / \Lambda^{2}\right), k_{\perp L u}=\ln \left(s_{q g} s_{g \bar{q}} / s \Lambda^{2}\right), k_{\perp L e}=\ln \left(s_{q g} s_{g \bar{q}} / s_{q \bar{q}} \Lambda^{2}\right)$, $s_{i j}=\left(p_{i}+p_{j}\right)^{2}$. The term $N_{q \bar{q}}\left(L, k_{\perp}\right)$ takes into account that the resolution of the gluon jet at a given $k_{\perp}$ implies restrictions on the phase space of the $q \bar{q}$ system. It can be obtained from $N_{q \bar{q}}(L)$ through the following relation: ${ }^{1}$

$$
N_{q \bar{q}}\left(L, k_{\perp}\right)=N_{q \bar{q}}\left(L^{\prime}\right)+\left(L-L^{\prime}\right) \cdot \frac{d}{d L^{\prime}} N_{q \bar{q}}\left(L^{\prime}\right) \text { with } L^{\prime}=k_{\perp}+c_{q} .
$$

As for massless jets all dynamical scales are given by the inter-jet angles, Eqs. (1), (2a,b) and (3) can be used to fit the ratio $C_{A} / C_{F}$ to the data. $N_{q \bar{q}}(L)$ is equivalent to the mean charge multiplicity of hadronic $e^{+} e^{-}$events as a function of the c.m. energy (measured by experiments ${ }^{10}$ and corrected not to include the contribution of $b \bar{b}$ events). As in this analysis no $b$-tagging procedure is applied to the data, an additive offset parameter $N_{0}$ is introduced to the fitting procedure $\left(N_{q \bar{q} g}=N_{q \bar{q} g}^{e x p}-N_{0}\right)$ to account for the extra multiplicity due to the decay of hadrons containing 


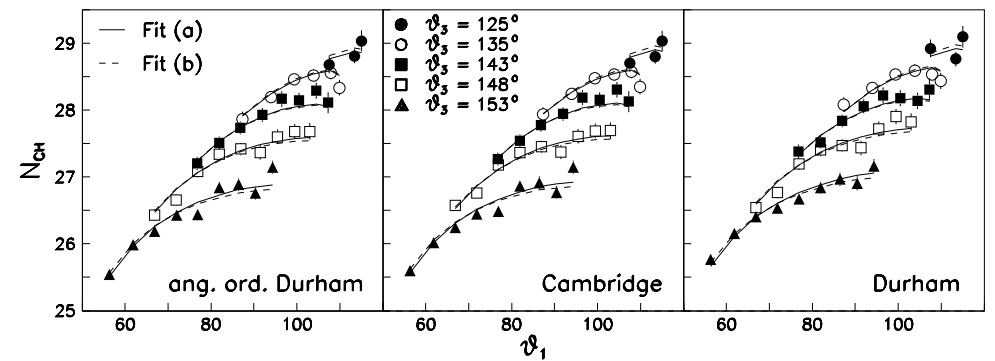

Figure 1. Mean charge multiplicity $N_{c h}$ in hadronic three-jet $\mathrm{Z}$ decays as a function of the event topology $\left(\theta_{1}, \theta_{3}\right)$ for three clustering algorithms. The curves are the results of the fits for two alternative predictions of Eqs. (2a) and (2b). See text.

Table 1. The results of the fits for both predictions and all clustering algorithms.

\begin{tabular}{|c|l|c|c|c|}
\hline pred. & jet finder & $C_{A} / C_{F}$ & $N_{0}$ & $\chi^{2} / n d f$ \\
\hline (2a) & Cambridge & $2.300 \pm 0.01$ & $0.69 \pm 0.03$ & 0.92 \\
(2a) & ang. ord. Durham & $2.329 \pm 0.03$ & $0.60 \pm 0.08$ & 1.03 \\
(2a) & Durham & $2.228 \pm 0.02$ & $0.97 \pm 0.03$ & 0.89 \\
\hline (2b) & Cambridge & $2.11 \pm 0.06$ & $0.36 \pm 0.08$ & 1.17 \\
(2b) & ang. ord. Durham & $2.151 \pm 0.04$ & $0.27 \pm 0.04$ & 1.33 \\
(2b) & Durham & $2.000 \pm 0.05$ & $0.60 \pm 0.06$ & 0.96 \\
\hline
\end{tabular}

the $b$ quark. The value of this parameter is expected to be of the order of $N_{0} \simeq 0.62^{11,12}$ and constant over a wide energy range. ${ }^{11}$

The gluon jet is not identified explicitly in this analysis. Therefore, the theoretical predictions for $N_{q \bar{q} g}$ at a given topology $\left(\theta_{3}, \theta_{2}\right)$ with the gluon jet being jet 1 , jet 2 or jet 3 are added with proper weights obtained from the QCD three-jet matrix element in the first order. Furthermore, the hadronization process affects the inter-jet angles by pulling close jets. Therefore, the corresponding correction calculated from the Ariadne Monte Carlo simulation by comparing the multiplicities defined at the partonic level with the ones "measured" at the hadronic level is taken into account.

Fig. 1 shows the measured mean charge multiplicities, $N_{c h}$, in hadronic three-jet $\mathrm{Z}$ decays as a function of $\theta_{1}$ for five $\theta_{3}$ intervals and three clustering algorithms. The results of the fits for both predictions and all clustering algorithms (see Table 1) agree well with the data shown in Fig. 1. The values of parameters fitted with Eq. (2a) are higher than those fitted with Eq. (2b) and mostly agree better with the expectation of $C_{A} / C_{F}=9 / 4$ and $N_{0} \simeq 0.62$. A weighted average of the values obtained for all clustering algorithms results in a value of $C_{A} / C_{F}=2.277 \pm 0.02 \pm 0.05$ for Eq. (2a) and $C_{A} / C_{F}=2.093 \pm 0.05 \pm 0.08$ for Eq. (2b) (the first error is statistical and the second one is systematic). One can see that the first value is more 


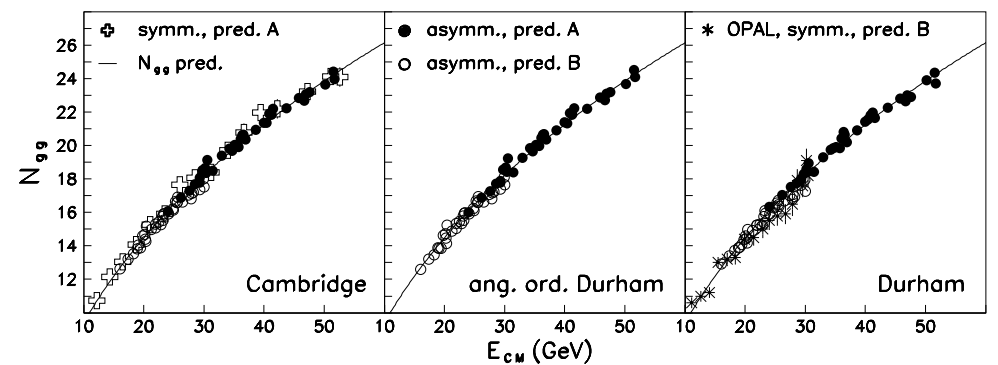

Figure 2. Mean charge multiplicity $N_{g g}\left(k_{\perp}\right)$ of a two-gluon colour-singlet system as a function of the effective c.m. energy $E_{c . m}$. given by $k_{\perp L e}$ for Eq. (2a) and $k_{\perp L u}$ for Eq. (2b). The curves show the prediction of Eq. (1). See text.

consistent with the QCD expectation.

Eqs. $(2 \mathrm{a}, \mathrm{b})$ can be used to extract the multiplicity $N_{g g}\left(k_{\perp}\right)$ from the measured values of $N_{c h}\left(\theta_{1}, \theta_{3}\right)$ shown in Fig. 1 . The results of this extraction (with fitted $N_{0}$ and fixed $C_{A} / C_{F}=9 / 4$ ) are shown in Fig. 2 for both predictions and all clustering algorithms over the effective c.m. energy range from 16 to $52 \mathrm{GeV}$. These results agree well with the prediction of Eq. (1) and with the previous DELPHI ${ }^{3}$ and OPAL ${ }^{4}$ results obtained only for symmetric three-jet $\mathrm{Z} \rightarrow q \bar{q} g$ decays.

In conclusion, the mean charge multiplicity in hadronic three-jet $\mathrm{Z}$ decays has been measured as a function of the event topology $\left(\theta_{1}, \theta_{3}\right)$. Fits of the two theoretical predictions to these data yield two values for the colour factor ratio $C_{A} / C_{F}$ of $2.277 \pm 0.02 \pm 0.05$ and $2.093 \pm 0.05 \pm 0.08$. Also the mean charge multiplicity of a two-gluon colour-singlet system has been extracted as a function of the effective c.m. energy, in good agreement with previous measurements for symmetric three-jet $\mathrm{Z}$ decays.

\section{References}

1. P. Edén, G. Gustafson, JHEP 9809, 015 (1998).

2. P. Edén, G. Gustafson, V. Khoze, Eur. Phys. J. C 11, 345 (1999).

3. DELPHI Collab., K. Hamacher et al., CERN-OPEN-2000-134.

4. OPAL Collab., G. Abbiendi et al., Eur. Phys. J. C 23, 597 (2002).

5. DELPHI Collab., Topology dependence of the charge multiplicity in hadronic three-jet events, ABS 247 contributed paper for ICHEP (Amsterdam, 2002).

6. DELPHI Collab., P. Abreu et al., Phys. Lett. B 449, 383 (1999).

7. Yu.L. Dokshitzer et al., JHEP 9708, 001 (1997).

8. S. Catani et al., Phys. Lett. B 269, 432 (1991).

9. CLEO Collab., M.S. Alam et al., Phys. Rev. D 46, 4822 (1992).

10. P.V. Chliapnikov, talk given at the 32nd ISMD (Alushta, 2002).

11. OPAL Collab., R. Akers et al., Phys. Lett. B 352, 176 (1995).

12. DELPHI Collab., P. Abreu et al., Phys. Lett. B 347, 447 (1995). 